\title{
RESEARCH
}

Open Access

\section{Loss of receptor tyrosine kinase-like orphan receptor 2 impairs the osteogenesis of mBMSCs by inhibiting signal transducer and activator of transcription 3}

Lizhen Lei $^{1,2}$, Zhuwei Huang ${ }^{1,2}$, Jingyi Feng ${ }^{1,2}$, Zijing Huang ${ }^{1,2}$, Yiwei Tao ${ }^{1,2}$, Xiaoli Hu ${ }^{1,2^{*}}$ and Xiaolei Zhang ${ }^{1,2^{*}}$

\begin{abstract}
Background: Receptor tyrosine kinase-like orphan receptor 2 (Ror2) plays a key role in bone formation, but its signaling pathway is not completely understood. Signal transducer and activator of transcription 3 (Stat3) takes part in maintaining bone homeostasis. The aim of this study is to reveal the role and mechanism of Ror 2 in the osteogenic differentiation from mouse bone marrow mesenchymal stem cells (mBMSCs) and to explore the effect of Stat3 on Ror2-mediated osteogenesis.
\end{abstract}

Methods: Ror2 CKO mice were generated via the Cre-loxp recombination system using Prrx1-Cre transgenic mice. Quantitative real-time PCR and western blot were performed to assess the expression of Stat3 and osteogenic markers in Ror2-knockdown mBMSCs (mBMSC-sh-Ror2). After being incubated in osteogenic induction medium for 3 weeks, Alizarin Red staining and western blot were used to examine the calcium deposit and osteogenic markers in Stat3 overexpression in mBMSC-sh-Ror2.

Results: Loss of Ror2 in mesenchymal or osteoblast progenitor cells led to a dwarfism phenotype in vivo. The mRNA expression of osteogenic markers (osteocalcin, osteopontin (OPN), and collagen I) in the ulna proximal epiphysis of Ror2 CKO mice was significantly decreased $(P<0.05)$. The mRNA and protein expression of Stat3 and osteogenic markers (Runx2, osterix, and OPN) decreased in mBMSC-sh-Ror2 cells $(P<0.05)$. The overexpression of Stat3 in mBMSC-sh-Ror2 cells rescued the calcium deposit and expression of Runx2, osterix, and OPN to a level comparable to normal mBMSCs.

Conclusions: Ror2 was essential for skeleton development by regulating mBMSCs' osteogenesis and osteoblast differentiation. Loss of Ror2 may impair the osteogenesis of mBMSCs by inhibiting Stat3.

Keywords: Ror2, Stat3, BMSCs, Osteogenesis

\footnotetext{
*Correspondence: huxiaol3@mail.sysu.edu.cn; zhangxl35@mail.sysu.edu.cn 'Guangdong Province Key Laboratory of Stomatology, Guangzhou 510080, Guangdong, China

Full list of author information is available at the end of the article
}

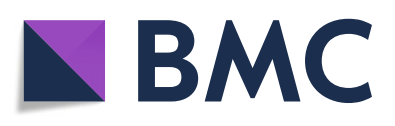

( ) The Author(s). 2020 Open Access This article is licensed under a Creative Commons Attribution 4.0 International License, which permits use, sharing, adaptation, distribution and reproduction in any medium or format, as long as you give appropriate credit to the original author(s) and the source, provide a link to the Creative Commons licence, and indicate if changes were made. The images or other third party material in this article are included in the article's Creative Commons licence, unless indicated otherwise in a credit line to the material. If material is not included in the article's Creative Commons licence and your intended use is not permitted by statutory regulation or exceeds the permitted use, you will need to obtain permission directly from the copyright holder. To view a copy of this licence, visit http://creativecommons.org/licenses/by/4.0/. The Creative Commons Public Domain Dedication waiver (http://creativecommons.org/publicdomain/zero/1.0/) applies to the data made available in this article, unless otherwise stated in a credit line to the data. 


\section{Background}

The proliferation and differentiation of mesenchymal stem cells toward osteoblasts is important for bone development and formation. The process involves in multiple regulatory signaling pathways and transcription factors [1, 2]. Both canonical and non-canonical Wnt signaling pathways play essential roles in mesenchymal stem cells differentiation into osteoblasts along with their proliferation and mineralization $[1,3,4]$.

The extracellular region of receptor tyrosine kinaselike orphan receptor 2 (Ror2) contains a cysteine-rich domain (CRD), which exhibits similarities to the CRDs found in the Frizzled (Fzd) family of seven transmembrane Wnt receptors [5-7]. Previous studies have identified Ror2 functions as a Wnt receptor/coreceptor regulated by canonical and non-canonical Wnt signaling pathways [8-12]. Mice with Ror2 homozygous mutation displayed shortened mandible, defects of derivatives of Meckel's cartilage, and notably shortening of the long bones of the appendicular skeleton [13, 14]. Ror2 mutation in humans accounts for recessive Robinow syndrome, which is characterized by macrocephaly, short stature, and mesomelic limb shortening, mostly in the forearms and brachydactyly $[15,16]$. The severe skeletal phenotypes in both Ror2 mutation mice and Robinow patients suggested a significant role of Ror2 in bone development and formation [11,13-16]. Available in vitro studies have proven that Ror2 can promote the osteogenic differentiation of mesenchymal stem cells $[3,17$, 18]. Nevertheless, direct evidence demonstrating the participation of Ror2 in osteogenesis of osteoblastic cell lineages in vivo is still required. The Ror2-mediated signaling pathways in osteogenesis of osteoblastic cell lineages are not completely understood. The Prrx1-Cre mice express Cre recombinase under the regulation of the paired related homeobox gene-1(Prx1)-derived regulatory element $[19,20]$. When Prrx1-Cre transgenic mice are crossed with a strain containing a loxp site-flanked sequence of interest, Cre-mediated recombination results in deletion of the floxed sequence in all mesenchyme-derived cells in the limbs and craniofacial tissue [19-22]. Therefore, in order to elucidate the role of Ror2 in osteogenesis of osteoblastic cell lineages in vivo, Ror $2^{\mathrm{f} / \mathrm{f}}$ transgenic mice which possess loxp sites flanking exons 3-4 of Ror2 gene [23] and the Prrx1-Cre transgenic mice were used to generate mice with Ror2 gene-specific deletion in mesenchymal progenitors.

The cytoplasmic transcription factor signal transducer and activator of transcription 3 (Stat3) activated by various cytokines and growth factors participated in cell proliferation, differentiation, and apoptosis [24, 25]. Activated Stat3 proteins translocated into the nucleus and then exerted transcriptional functions, thus taking part in tumorigenesis, self-renewal, and pluripotency of stem cells [26]. Mice with selective disruption of Stat3 in osteoblasts or osteocytes showed reduced bone formation [27, 28]. High level of Ror2 expression in platinumresistant ovarian tumor was found to be coupled with upregulation of Stat3, suggesting that Stat3 might act as the signaling downstream of Ror2 [29]. IL-6 induced the transcription of Ror2 to accelerate osteoblast-like differentiation and calcification in human adipose tissuederived mesenchymal stem cell (hADSCs), while suppression of Stat3 in hADSCs inhibited IL-6-mediated Ror2 expression and mineralization [30]. However, the role of Stat3 in Ror2-mediated osteogenesis of mBMSCs has not been unveiled.

In this study, we investigated the role of Ror2 in embryonic and neonatal bone formation though transgenic mice of limb bud mesenchyme and craniofacial mesenchymespecific Ror2 deficiency. In addition, the effect of Stat3 on Ror2-mediated osteogenesis of mBMSCs was explored in vitro.

\section{Methods \\ Mouse lines}

Mice were used according to federal guidelines and as approved by the Animal Ethical and Welfare Committee of Sun Yat-sen University (approval number SYSUIACUC-2018-000275). Mice were described in the literature and purchased from the Jackson Laboratory: Prrx1Cre (stock no. 005584) and Ror2 ${ }^{\mathrm{f} / \mathrm{f}}$ (stock no. 018354) $[20,23]$. To generate Ror2 conditional knockout mice, $\operatorname{Ror}^{\mathrm{f} / \mathrm{f}}$ mice were first crossed with Prrx1-Cre transgenic mice to obtain Prrx1-Cre; Ror2 ${ }^{\mathrm{f} /+}$ mice. Then, Prrx1Cre; Ror $2^{\mathrm{f} /+}$ mice were crossed with Ror $2^{\mathrm{f} / \mathrm{f}}$ mice to get Prrx1-Cre; Ror2 ${ }^{\mathrm{f} / \mathrm{f}}$ (hereafter called Ror2 CKO) mice. For embryos or neonatal mice, both male and female were used in the analyses as sex could not be clearly identified in embryos or neonatal mice. Three or more littermate mice per group were examined, and representative images were shown in Fig. 1. Genotyping was conducted by polymerase chain reaction (PCR) of tail lysate. Primer sequences were provided in Table 1.

\section{Skeletal staining}

According to the standard protocol, skeletons from embryonic (E14.5) and neonatal (P0) mice were processed for Alizarin Red and Alcian Blue staining to visualize bone and cartilage [31].

\section{Histology and immunohistofluorescence (IHF) staining}

Forelimbs of P0 newborn mice were isolated, fixed overnight in $4 \%$ paraformaldehyde (PFA, Servicebio, Wuhan, China), and were embedded in optimal cutting temperature (OCT) compound (Tissue-Tek). Ulna longitudinal sections $(8 \mu \mathrm{m})$ using the elbow joint as landmark were cut and stained for both histology and IHF staining. 


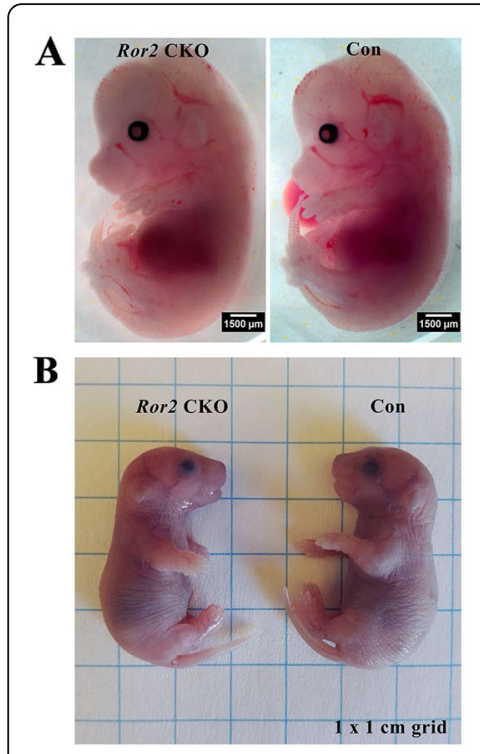

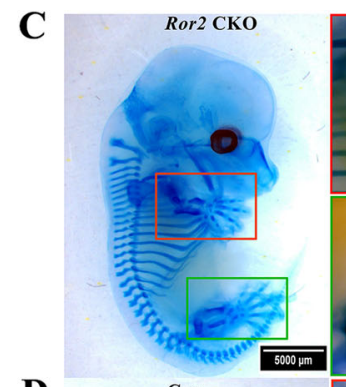

D

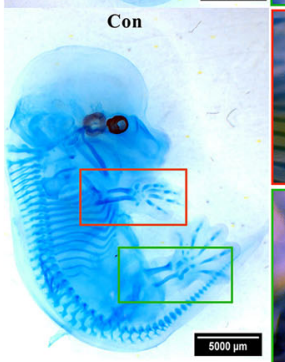

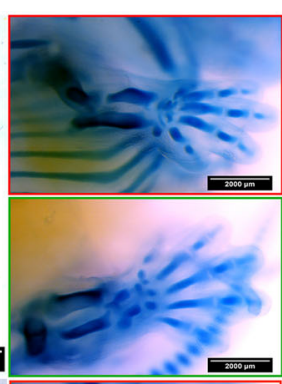

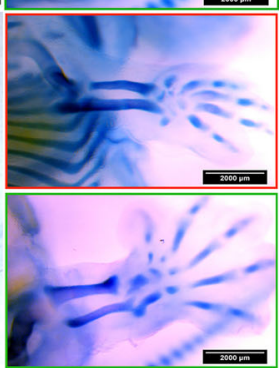

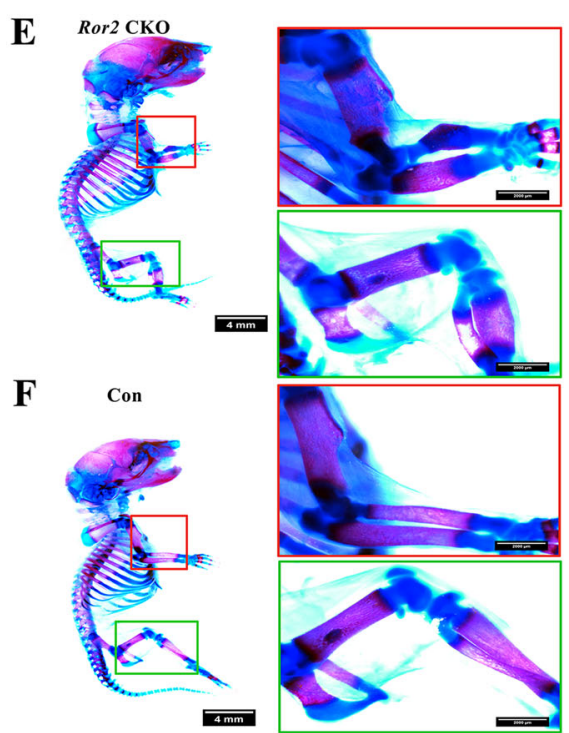

Fig. 1 The dwarfism phenotype of Ror2 CKO mice. Compared with the littermate control (Con) mice, the limbs were significantly shortened in Prrx1-Cre; Ror2 ${ }^{\mathrm{f} / \mathrm{f}}$ (Ror2 CKO) mice at both embryonic (E14.5) and neonatal (PO) stage. a, b Ex vivo observation of Ror2 CKO and control mice at E14.5 (a) and P0 (b), scale bar $=1500 \mu \mathrm{m}$. c-f Whole-mount skeletal staining of Ror2 CKO and Con mice at E14.5 (c, d), scale bar $=5000 \mu \mathrm{m}$, and PO (e, f), scale bar $=4 \mathrm{~mm}$, respectively. The boxed region is enlarged in the right panel, scale bar $=2000 \mu \mathrm{m}$

To assess the general histology and mineralized tissue in the region of interest, the histological stainings including hematoxylin-eosin (H\&E) and Von Kossa were applied.

Using a standard protocol, IHF staining was performed with the appropriate primary antibodies and secondary antibodies (Table 2). Cryostat sections were mounted in mounting medium with DAPI from Vector Laboratories (H-1200). The images were captured by an inversion fluorescence microscope (Zeiss, Oberkochen, Germany).

\section{Cell culture}

Mouse bone marrow mesenchymal stem cells (mBMSCs) isolated from the bone marrow of $\mathrm{C} 57 \mathrm{BL} / 6$ mice were provided by Cyagen Biosciences, Inc. (Guangzhou, China). Identification of the cells according to the cell surface phenotypes and multipotency was performed by the supplier. mBMSCs were cultured in alpha-MEM (Gibco) supplemented with $10 \%$ fetal bovine serum (FBS, Gibco) and incubated at $37^{\circ} \mathrm{C}$ in a humidified atmosphere of $5 \% \mathrm{CO}_{2}$. mBMSCs in passages 6-9 were used in this study.

\section{Lentiviral vector construction and transduction}

The recombinant lentivirus vector downregulation of mouse Ror2 gene and overexpression of mouse Stat3 gene were generated by Cyagen Biosciences, Inc. (Guangzhou, China) and iGeneBio Biotechnology (Guangzhou, China), respectively.

According to the manufacturer's instructions, mBMSCs were infected with lentivirus-based shRNA vector downregulation of Ror2 (sh-Ror2) and lentivirusbased shRNA vector overexpression of Stat3 (sh-Stat3).

Table 1 Primers for PCR and qRT-PCR

\begin{tabular}{llll}
\hline Gene & Forward & Reverse & Application \\
\hline Prrx1 & GCG GTC TGG CAG TAA AAA CTA TC & GTG AAA CAG CAT TGC TGT CAC TT & PCR \\
Ror2 & TGC AGG TIT TGA GCC CTA AC & CGA GAA TGA CTT CCC TGT CC & PCR \\
GAPDH & CCTTCCGTGTTCCTACCC & CAACCTGGTCCTCAGTGTAG & qRT-PCR \\
Ror2 & ACGCAATGTGCTGGTGTAC & TGTCAGAGTCGATGGAGAAC & qRT-PCR \\
Stat3 & CATCCTGAAGCTGACCCAGG & TATTGCTGCAGGTCGTGGT & qRT-PCR \\
Runx2 & CCGTCACCTCCATCCTCTTC & AATACGCATCACAACAGCCACA & qRT-PCR \\
OSX & ACCCTTCCCTCACTCATTTCCTG & TGCCTTGTACCACGAGCCATAG & qRT-PCR \\
OPN & CTCCAATCGTCCCTACAGTCG & CCAAGCTATCACCTCGGCC & qRT-PCR \\
COL1A1 & CCCAAGGAAAAGAAGCACGTC & ACATTAGGCGCAGGAAGGTCA & qRT-PCR \\
OCN & GCTGCCCTAAAGCCAAACTCT & AGAGGACAGGGAGGATCAAGTTC & qRT-PCR \\
\hline
\end{tabular}


Table 2 Antibodies used for immunoblotting

\begin{tabular}{|c|c|c|}
\hline Marker (species) & Dilution & Distributor/source (catalog number) \\
\hline \multicolumn{3}{|l|}{ Primary antibody } \\
\hline RANKL mouse mAb & $1: 100$ & Novus Biologicals (12A380) \\
\hline OSX rabbit mAb & $1: 70(\mathrm{IHF})$ & Santa Cruz (A13) \\
\hline OSX rabbit mAb & $1: 1000(\mathrm{WB})$ & Abcam (ab22552) \\
\hline OPN goat mAb & $1: 200(\mathrm{IHF})$ & R\&D (af808) \\
\hline OPN rabbit pAb & $1: 1000(\mathrm{WB})$ & ZEN BIO(380437) \\
\hline COL1A1 goat pAb & $1: 200$ & Santa Cruz (sc-8784-R) \\
\hline CD31 rabbit mAb & $1: 200$ & Biolegend (102502) \\
\hline SOX9 rabbit mAb & $1: 300$ & Millipore (ab-5535) \\
\hline Ror2 rabbit pAb & $1: 1000$ & CST (4105) \\
\hline Stat3 mouse mAb & $1: 1000(\mathrm{WB})$ & CST (9139) \\
\hline Stat3 mouse mAb & 1:100(ICF) & CST (9139) \\
\hline Runx2 rabbit mAb & $1: 1000$ & CST (8486) \\
\hline pStat3 mouse mAb & $1: 2000$ & CST (4113) \\
\hline \multicolumn{3}{|l|}{ Secondary antibody } \\
\hline Anti-rabbit lgG (Fluor ${ }^{\circledR} 488$ Conjugate) & 1:500 (IHF) & Yeasen Biotech (33106ES60) \\
\hline Anti-mouse lgG (Fluor 568 Conjugate) & 1:500 (IHF) & Abcam (ab175473) \\
\hline Anti-rat IgG (Fluor 594 Conjugate) & 1:500 (IHF) & Yeasen Biotech (34412ES60) \\
\hline Anti-mouse lgG (Fluor 564 Conjugate) & $1: 300$ (ICF) & Yeasen Biotech (33212ES60) \\
\hline Anti-mouse IgG HRP-linked Ab & $1: 2000$ (WB) & CST (7076) \\
\hline Anti-rabbit lgG HRP-linked Ab & 1:2000 (WB) & CST (7074) \\
\hline
\end{tabular}

IHF immunohistofluorescence, WB western blot, ICF immunocytofluorescence

Briefly, mBMSCs were plated at $1.5 \times 10^{4} \mathrm{cells} / \mathrm{cm}^{2}$ in 12 well plates overnight and then infected with lentivirus in the presence of $5 \mu \mathrm{g} / \mathrm{mL}$ polybrene (iGeneBio Biotechnology, Guangzhou, China) for $8 \mathrm{~h}$. After $72 \mathrm{~h}, \mathrm{mBMSCs}$ infected with sh-Ror2 were selected with $1 \mu \mathrm{g} / \mathrm{mL}$ puromycin (Solarbio, Beijing, China). Those mBMSCs infected with sh-Ror2 and sh-Stat 3 were selected with $1 \mu \mathrm{g} / \mathrm{mL}$ puromycin and $50 \mu \mathrm{g} / \mathrm{mL}$ hygromycin B (Solarbio, Beijing, China). The expression of Ror2 and Stat3 was assessed by quantitative real-time polymerase chain reaction (qRTPCR) and western blot analysis.

\section{Osteogenic differentiation and calcium deposition determination}

For osteogenic differentiation, the cells were plated at $2 \times 10^{4}$ cells $/ \mathrm{cm}^{2}$ in 6 -well plates and cultured in $2 \mathrm{ml}$ of alpha-MEM containing 10\% FBS. When cells reached approximately $60-70 \%$ confluence, they were switched to the osteogenic induction medium, which consisted of alpha-MEM supplemented with $10 \%$ (vol/vol) FBS, $10^{-7} \mathrm{M}$ dexamethasone (Sigma, USA), $10 \mathrm{mM} \beta$-glycerol phosphate (Solarbio, Beijing, China), and $50 \mu \mathrm{M}$ ascorbate-2phosphate (Solarbio, Beijing, China) for 3 weeks. The calcium deposition was assessed by staining with $40 \mathrm{mM}$ Alizarin Red S solution (Cyagen Biosciences, Guangzhou,
China) at room temperature for $15 \mathrm{~min}$. The Alizarin Red S concentrations were determined by a quantitative destaining procedure using 10\% cetylpyridinium chloride (CPC) (Sigma, USA) for $15 \mathrm{~min}$ at room temperature. The absorbance value at $562 \mathrm{~nm}$ was then measured by microplate spectrophotometer (Bio-Tek, UK).

RNA isolation, reverse transcription, and qRT-PCR analysis Total RNA of mouse forelimbs and mBMSCs was extracted using an Eastep Super total RNA extraction kit (Promega, Shanghai, China) according to the manufacturer's instructions and then was reverse transcribed using a PrimeScriptTM RT Master Mix (Takara Bio, Ohtsu, Japan). The quantification was performed using the TB Green ${ }^{\mathrm{TM}}$ Premix Ex Taq $^{\mathrm{TM}}$ II (Tli RNaseH Plus) reagent (Takara Bio, Ohtsu, Japan). The relative expression of target gene was normalized in relative to the level of GAPDH using the $2^{-\Delta \Delta C T}$ method. Primer sequences were provided in the Table 1.

\section{Western blot}

Total protein and nucleoprotein lysates from cells were extracted using the RIPA lysis buffer (CWBIOTECH, Beijing, China) according to the manufacturer's instructions. The proteins were separated by sodium dodecyl sulfate- 
polyacrylamide gel electrophoresis (SDS-PAGE, Genscript, Nanjing, China) and transferred to a polyvinylidene fluoride (PVDF) membrane (Millipore, Bedford, MA, USA). The membrane was then blocked with 5\% bovine serum albumin (BSA, Biofroxx, Germany) for $2 \mathrm{~h}$, incubated with relevant primary antibodies (Table 2) at $4{ }^{\circ} \mathrm{C}$ overnight, and then incubated with corresponding secondary antibodies (Table 2) for $1 \mathrm{~h}$ at room temperature. Bands were detected with a chemiluminescence kit (Millipore, Bedford, MA, USA).

\section{Immunocytofluorescence (ICF) staining}

Approximately $0.2 \times 10^{4}$ cells $/ \mathrm{cm}^{2}$ cells were seeded in 24-well plates with cover slides. When reached approximately $60 \%$ confluence, they were switched to the osteogenic induction medium for $24 \mathrm{~h}$. The cells were fixed in $4 \%$ PFA for $10 \mathrm{~min}$ and then permeabilized in $0.1 \%$ Triton X-100 (CWBIOTECH, Beijing, China) for $5 \mathrm{~min}$ at room temperature. Next, the cells were blocked with $5 \%$ BSA at room temperature for $1 \mathrm{~h}$ and then incubated with Stat 3 antibodies (Table 2) at $4{ }^{\circ} \mathrm{C}$ overnight. After a PBS wash for $15 \mathrm{~min}$, the cells were incubated with corresponding secondary antibodies (Table 2) diluted in 5\% BSA at room temperature for $1 \mathrm{~h}$. Finally, the cells were washed for $15 \mathrm{~min}$ with PBS, followed by nuclear staining with DAPI (Telenbiotech, Guangzhou, China). Confocal laser scanning fluorescence microscopy (Nikon Eclipose Ni-E, Japan) was employed to analyses Stat3 staining in cells.

\section{Statistical analysis}

The data were presented as means \pm standard deviation (SD). Comparisons among groups were performed by one-way ANOVA followed by Bonferroni's post hoc test with GraphPad Prism 7.0 software. The significance was set at $P<.05$.

\section{Results}

The dwarfism phenotype of Ror2 CKO mice

The embryonic (E14.5) and neonatal (P0) Ror2 CKO mice displayed dwarfism where shortened bone was found in limbs (Fig. 1a, b). Whole-mount skeletal staining by Alizarin Red and Alcian Blue showed that the length of forelimb and hindlimb was distinctly reduced in Ror2 CKO mice (Fig. 1c, e) when compared with littermate controls (Fig. 1d, f).

\section{Impaired osteogenesis in neonatal Ror2 CKO mice}

Remarkably reduced mineralization in ulna proximal epiphysis was detected by Von Kossa staining in Ror2 CKO mice (Fig. 2). IHF staining and qRT-PCR were performed with ulna proximal epiphysis of PO mice. IHF staining showed that the osteogenic differentiation markers osterix (OSX), osteopontin (OPN), collagen I (COL1A1), and Sox9 were dramatically diminished in Ror2 CKO mice (Fig. 3a). Consistent with IHF staining results, the mRNA expression of osteocalcin $(\mathrm{OCN})$, OPN, and COL1A1 was significantly reduced in Ror 2 CKO mice compared with controls $(P<0.05$; Fig. 3b).

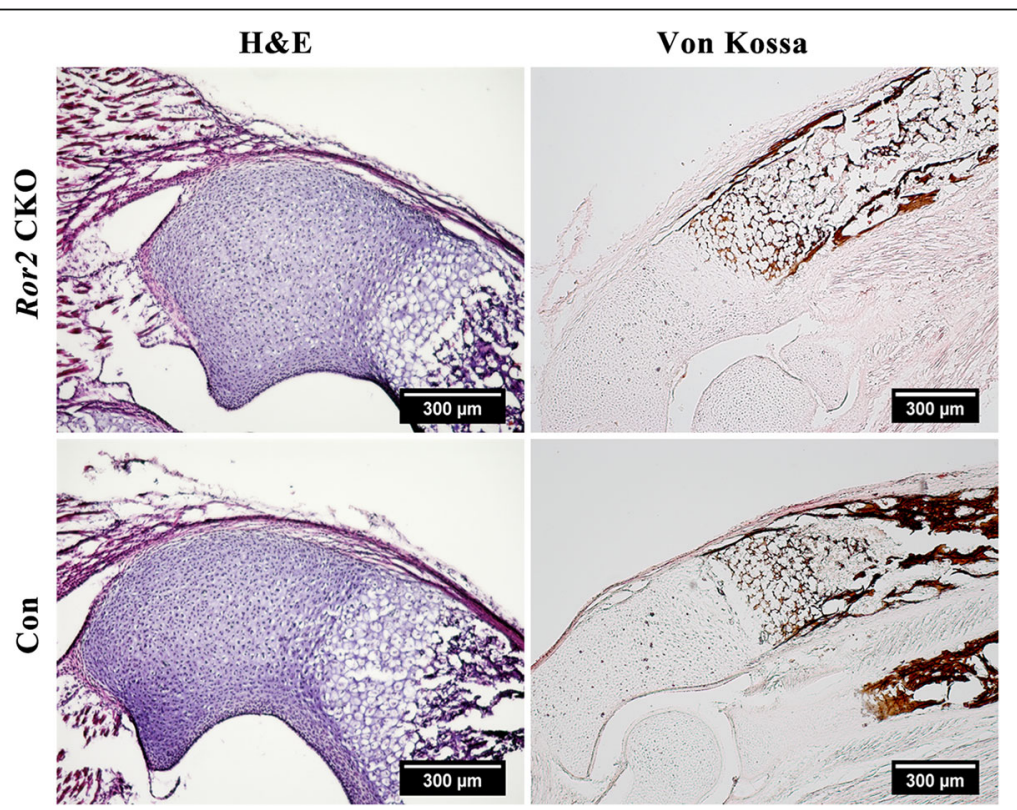

Fig. 2 Histology staining of ulna proximal epiphysis presented an impaired osteogenesis in neonatal Ror2 CKO mice (P0) compared with the littermate control (Con). Hematoxylin-eosin (H\&E) and Von Kossa staining were used to observe the general histology and mineralized tissue, respectively. Scale bar $=300 \mu \mathrm{m}$. Compared with the control, mineralized tissue visualized in brown by Von Kossa staining was significantly decreased in Ror2 CKO mice 


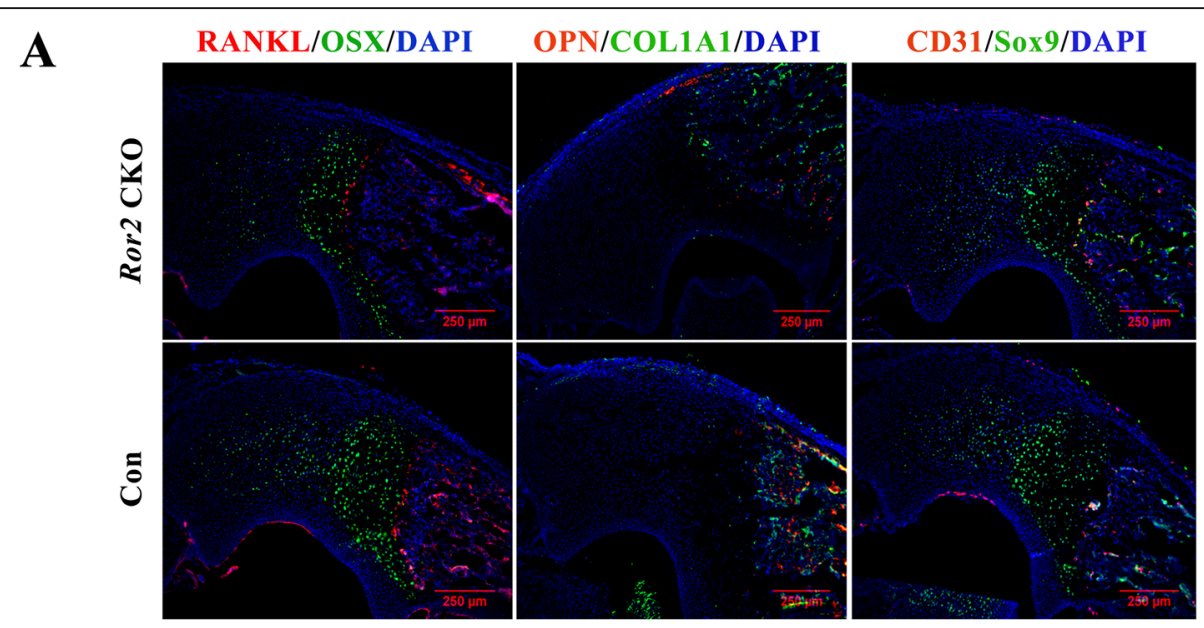

B

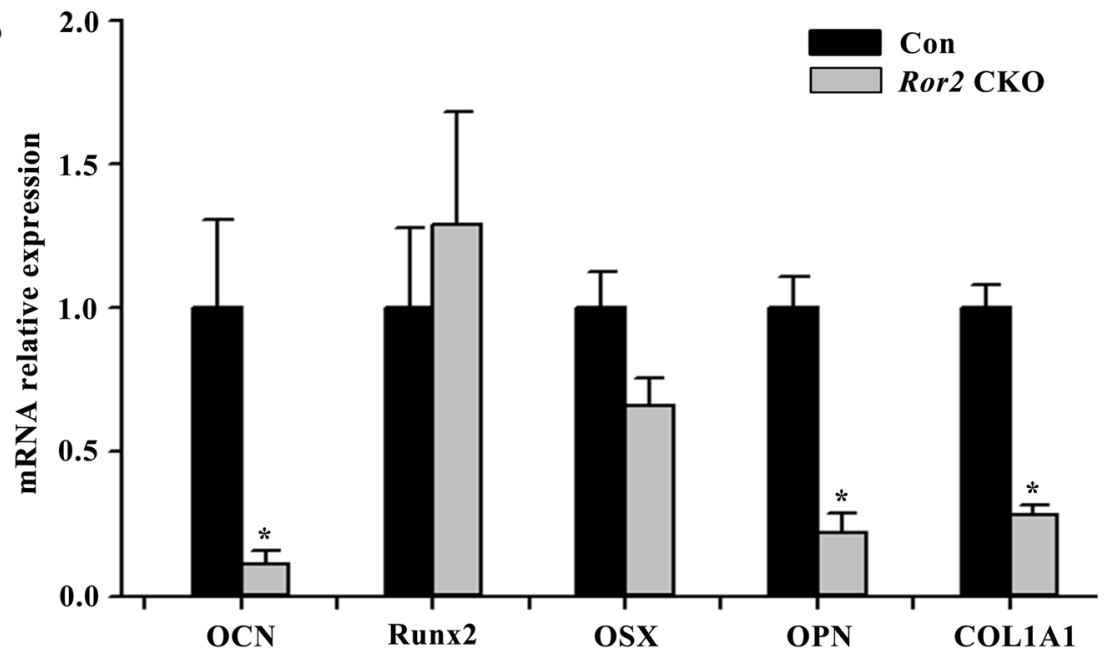

Fig. 3 Reduced expression of osteogenic markers in neonatal Ror2 CKO mice (PO) compared with the littermate control (Con). a Immunohistofluorescence staining of ulna proximal epiphysis showed that less OSX, OPN, COLIA1, and Sox9 were found in Ror2 CKO samples. Scale bar $=250 \mu \mathrm{m}$. b RNAs were extracted from ulna proximal epiphysis. Quantitative real-time PCR revealed a significant decrease of mRNA expression of OCN, OPN, and COLIA1 in Ror2 CKO compared with controls ( $n=3,{ }^{*} P<0.05$ vs. Con)

\section{Knockdown of Ror2 in mBMSCs led to reduced} osteogenic markers and Stat3 expression

Transfected with recombinant lentivirus vectors, the downregulation of Ror2 into mBMSCs was performed to investigate the effect of Ror2 on osteogenic differentiation from mBMSCs (Fig. 4a-d). After osteogenic induction for 3 weeks, less calcium deposit was observed in the mBMSC-sh-Ror2 cells when compared with mBMSC-mock1 cells examined by Alizarin Red staining (Fig. 6a) and quantitative Alizarin Red concentration measurements (Fig. 6b). The western blot results revealed that knockdown of Ror2 significantly suppressed the expression of Runx2, OSX, and OPN in mBMSCs in response to osteogenic induction $(P<0.05$; Fig. $7 \mathrm{a}, \mathrm{b})$. No significant difference between mBMSC and mBMSC-mock1 was observed. Meanwhile, the expression of total Stat3 was significantly decreased in mBMSC-sh-Ror2 cells after osteogenic induction determined by qRT-PCR and western blot $(P<0.05$; Fig. $5 \mathrm{a}-\mathrm{c})$.

Furthermore, ICF also showed that in comparison with mBMSC-mock1 cells, knockdown of Ror2 in mBMSCs caused a decrease of intracellular accumulation of Stat3 (Fig. 5d). At the same time, nuclear phosphorylated Stat3 (pStat3) was obviously reduced in mBMSC-sh-Ror2 cells after osteogenic induction for $24 \mathrm{~h}$ monitored by western blot (Fig. 5e).

\section{Reduced osteogenic differentiation in Ror2-knockdown} mBMSCs was restored by overexpression of Stat3

In Ror2-knockdown mBMSCs, overexpression of Stat3 was conducted through utilizing recombinant lentivirus vectors (Fig. 5f, g). Alizarin Red staining demonstrated 


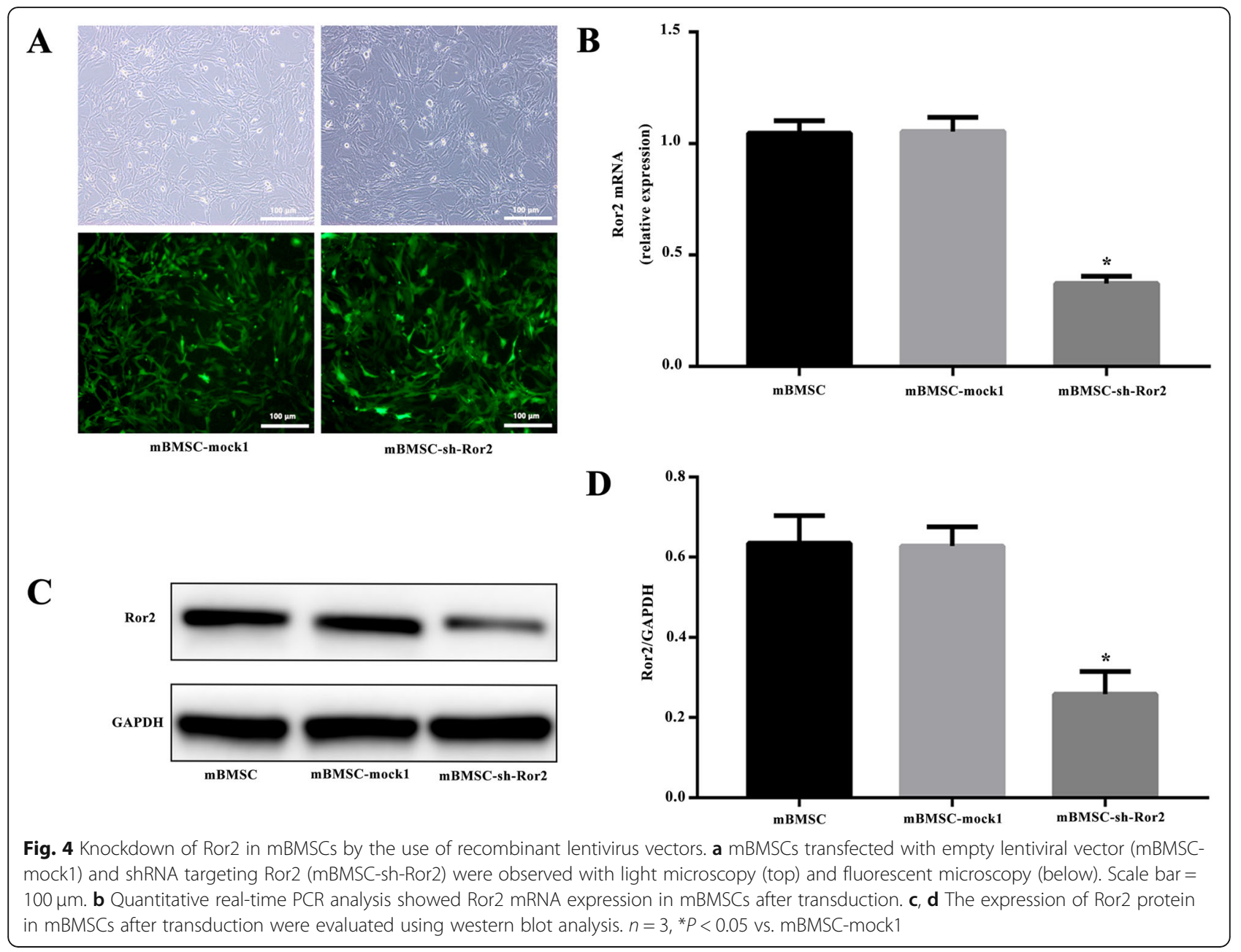

that similar amount of calcium deposits was observed in the mBMSC-sh-Ror2-Stat3 cells when compared with controls (mBMSC-mock1 and mBMSC-mock2) (Fig. 6a). Overexpression of Stat3 in mBMSC-sh-Ror2 cells caused a recovery of osteogenic ability to a level comparable to controls (mBMSC-mock1 and mBMSC-mock2) after 3 weeks of osteogenic induction, which was proven by the increased expression of osteogenic markers (Runx2, OSX, and OPN) (Fig. 7a, b). Thus, it was logical to speculate that Ror2 might facilitate osteogenic differentiation through Stat3.

\section{Discussion}

In the present study, a significantly short limb phenotype was observed on Ror2 CKO mice, where Ror2 was specifically knock out from limb bud mesenchyme and craniofacial mesenchyme. In comparison with the global Ror2 knockout mice which were neonatal dead due to forced respiration and severe cyanosis [11, 14], no difference of survival rate and lifespan was found between the Ror2 CKO mice and their littermate siblings. In the observation period of 4 postnatal weeks, the Ror2 CKO mice were alive with more conspicuous dwarfism over time. In our study, loss of Ror2 in limb bud mesenchyme resulted in considerably reduced mineralization in ulna proximal epiphysis. Our observation was in line with that of Takeuchi et al., which reported that a lack of calcification were observed in enchondral ossification centers when Ror2 was disrupted in mice [14]. The diminished expression of osteogenic markers in our study indicated that deletion of Ror2 in limb mesenchyme indeed impaired the ossification. As known, OCN and OPN, which are produced during bone formation and late in the mineralization process, play synergistic roles in determining bone size and shape [32]. The decreased expression of OCN and OPN in Ror2 CKO mice indicated that Ror2 might be necessary for bone formation and morphology. In addition, during limb development, Sox9 was involved in the determination of both osteogenic and chondrogenic cell lineages. Neither mesenchymal condensations nor osteoblast lineage and its differentiation was observed in early limb buds of Sox 9 depletion [33, 34]. Thus, the effect of Ror2 on bone formation might partly rely on regulating Sox9 signaling. 


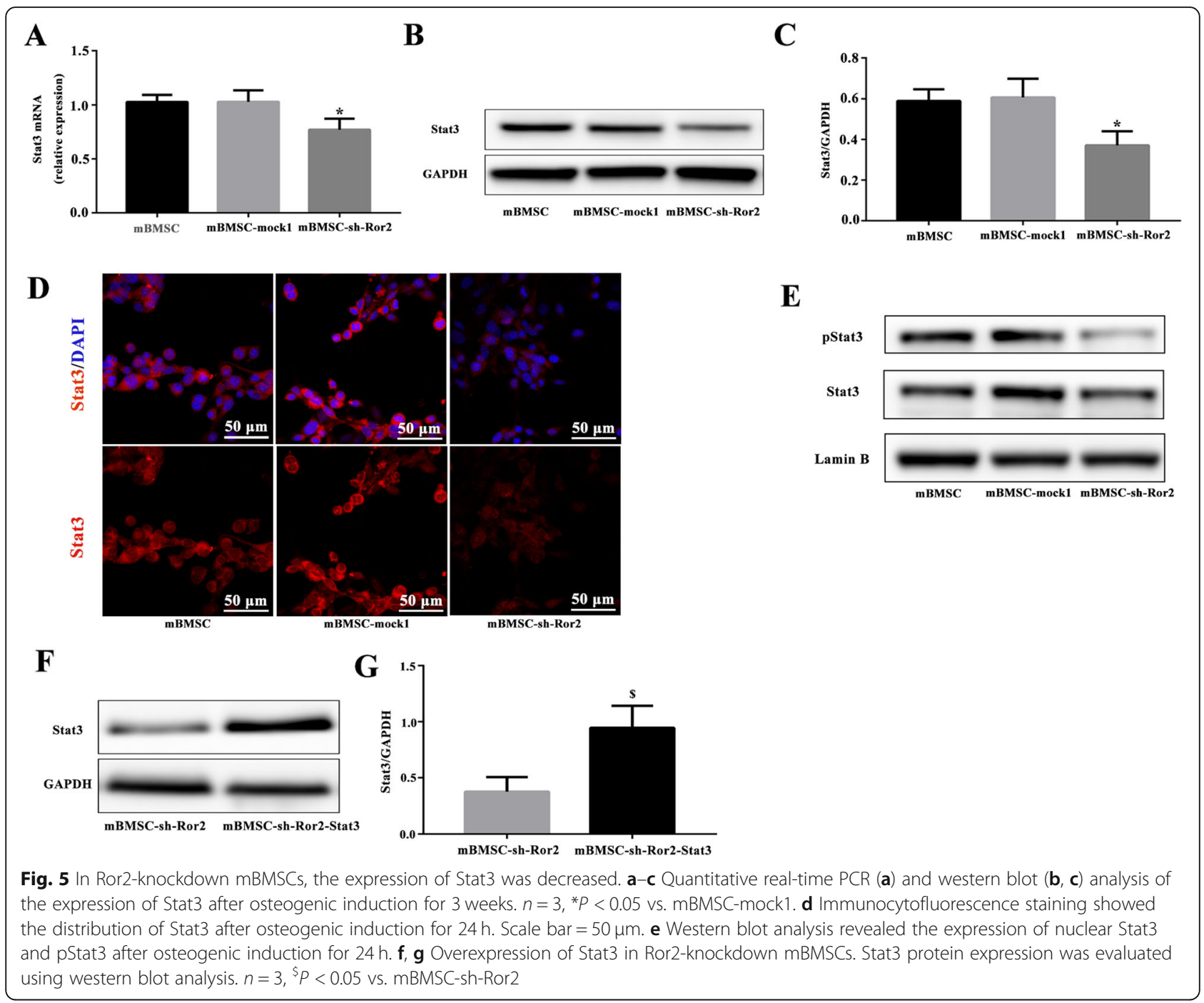

To gain insight into the cellular mechanisms of Ror2mediated osteogenesis, we studied the effect of downregulation of Ror2 on osteogenic differentiation of mBMSCs in vitro. Available literature reported that the Ror2 expression was increased during early stages of osteoblast differentiation and was peaked in committed proliferating preosteoblasts, contributing to osteoblastogenesis $[3,10,17]$. Runx 2 and OSX are expressed in preosteoblasts and play essential roles in the differentiation of preosteoblasts to immature osteoblasts [35]. In our study, when Ror2 was knockdown in mBMSCs, the osteogenic markers of mBMSCs, including Runx2, OSX, and OPN, were significantly decreased, which suggested the inhibition effect of Ror2 knockdown on mBMSCs' osteogenesis. Our results were in accordance with previous reports, in which the overexpression of Ror2 in mesenchymal stem cells promoted the formation of mineralized extracellular matrix and expression of osteogenic transcription factors, whereas the downregulation of Ror2 resulted in the opposite [17, 36]. In addition, in preosteoblast cell line MC3T3-E1 and a mouse calvariae ex vivo organ culture model, Ror2 also strongly promoted matrix mineralization and increased bone formation [17, 37]. Collectively, it was proven that Ror2 played a promotive role in osteogenesis.

The expressions of Stats family have been found in bone tissues, which were believed to take part in activities of both osteoblasts and osteoclasts [24]. The increased bone mass and accelerated healing of bone fracture were observed in Stat1-deficient mice [38, 39]. The mechanism might be that Stat1 negatively regulated osteoblast differentiation by suppressing transcription of Runx 2 and OSX. Stat 4 and Stat 6 were found involved in osteoclastogenesis and arthritis [40, 41]. Notably, Stat 3 took part in a series of biological process of the bone, including cell growth, apoptosis, and motility. Furthermore, Stat3 was believed to be most important in osteoblasts and osteoclasts compared with other Stats [24]. Ror2 promoter region 

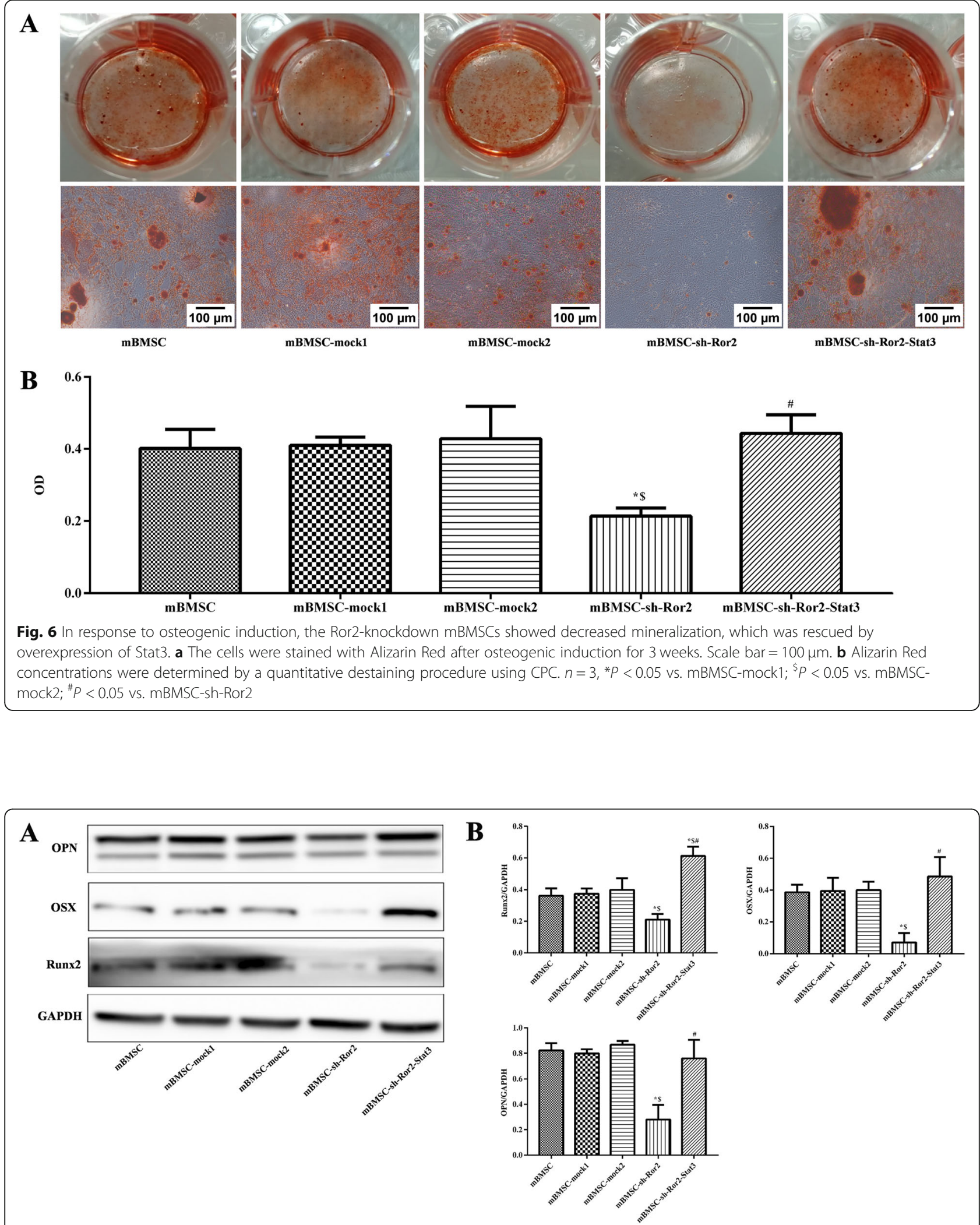

Fig. 7 In response to osteogenic induction, the less expressed osteogenic makers in Ror2-knockdown mBMSCs were restored by overexpression of Stat3. a $\mathbf{b}$ Western blot analysis of the expression of osteogenic specific markers after osteogenic induction for 3 weeks. $n=3$, ${ }^{*} P<0.05$ vs. mBMSC-mock1; ${ }^{\$} P<0.05$ vs. mBMSC-mock2; ${ }^{\prime} P<0.05$ vs. mBMSC-sh-Ror2 
contained a GAS motif, which is speculated as a cis element for Stats [29, 42]. Inhibition of Stat3 in hADSCs reduced the ability of IL- 6 to induce Ror2 mRNA expression and osteoblast-like differentiation and calcification [30]. These findings hinted a question that whether or not Stat3 was involved in the Ror2-mediated osteogenic differentiation of mBMSCs. In the current study, a significant decrease of Stat3 expression was observed in Ror2knockdown mBMSCs in response to osteogenic induction at both mRNA and protein levels. Except cytokine receptors associated with JAKs, Stat3 can be activated by receptor tyrosine kinases and G protein-coupled receptors [43]. Upon ligand binding, Ror2 was found to form receptor complex with other components, including Src and CK1ع, then stimulates Stat3 activation, ultimately leading to cell invasion [44]. In our study, the overexpression of Stat3 in Ror2-knockdown mBMSCs rescued the decreased osteogenic differentiation of Ror2-knockdown mBMSCs. A possible explanation might be that the intracellular accumulation of Stat 3 could promote the osteogenic gene transcription in mBMSCs cultured in osteogenic induction medium. However, whether or not Ror2 directly interacts with Stat3 in mBMSCs' osteogenesis requires further study.

\section{Conclusions}

Taken together, our study suggested a potential role and mechanism of Ror2 in mBMSCs' osteogenic differentiation. Ror2 and Stat3 were essential for skeleton development by regulating mBMSCs' osteogenesis and osteoblast differentiation. The impaired osteogenesis by knockdown of Ror2 was rescued by increased Stat 3 .

\section{Supplementary information}

Supplementary information accompanies this paper at https://doi.org/10. 1186/s13287-020-01646-2.

Additional file 1: Figure S1. The PCR results of genotyping Ror2 CKO mice. The protocol of genotyping was performed according to the Jackson Lab instructions. Lane 1: blank control (no DNA), Lane 2: negative control (DNA from wild-type mouse), Lane 3: Prrx1-cre; Ror2 ${ }^{c /+}$, Lane 4: Prrx1-Cre; Ror2 ${ }^{c / c}$ (e.g. Ror2 CKO), Lane 5: Ror2 ${ }^{c /+}$, Lane 6: Ror2 ${ }^{c / c}$. Figure S2. The mBMSCs characterizations by flow cytometry.

\footnotetext{
Abbreviations

Ror2: Receptor tyrosine kinase-like orphan receptor 2; Stat3: Signal transducer and activator of transcription 3; BMSCs: Bone marrow mesenchymal stem cells; OPN: Osteopontin; hADSCs: Human adipose tissue-derived mesenchymal stem cells; CKO: Conditional knockout; PCR: Polymerase chain reaction; IHF: Immunohistofluorescence; H\&E: Hematoxylin-eosin; mBMSCs: Mouse bone marrow mesenchymal stem cells; qRTPCR: Quantitative real-time polymerase chain reaction; FBS: Fetal bovine serum; BSA: Bovine serum albumin; ICF: Immunocytofluorescence; OSX: Osterix; COL1A1: Collagen I; OCN: Osteocalcin; Stats: Signal transducers and activators of transcription
}

Acknowledgements

Not applicable.

\section{Authors' contributions}

LZL and XLZ designed the experiments. LZL, JYF, and ZJH performed the experiments. LZL, ZWH, and YWT analyzed the data. LZL wrote the manuscript. XLH and XLZ participated in the critical revision of the paper. All authors read and approved the final manuscript.

\section{Funding}

This work was supported by the National Natural Science Foundation of China (grant no. 11772361, 81470731).

\section{Availability of data and materials}

All datasets used and/or analyzed during the current study are available from the corresponding author on reasonable request.

\section{Ethics approval and consent to participate}

Mice were used according to federal guidelines and as approved by the Animal Ethical and Welfare Committee of Sun Yat-sen University (approval number SYSU-IACUC-2018-000275).

\section{Consent for publication}

Not applicable.

\section{Competing interests}

The authors declare that they have no competing interests.

\section{Author details}

'Guangdong Province Key Laboratory of Stomatology, Guangzhou 510080, Guangdong, China. ${ }^{2}$ Department of Operative Dentistry and Endodontics, Guanghua School and Hospital of Stomatology, Sun Yat-sen University, Guangzhou 510055, Guangdong, China.

Received: 21 January 2020 Revised: 20 February 2020 Accepted: 10 March 2020 Published online: 26 March 2020

\section{References}

1. Gonciulea A, de Beur SJ. The dynamic skeleton. Rev Endocr Metab Disord. 2015;6:79-91.

2. Long F. Building strong bones: molecular regulation of the osteoblast lineage. Nat Rev Mol Cell Biol. 2011;13:27-38.

3. Boland GM, Perkins G, Hall DJ, Tuan RS. Wnt3a promotes proliferation and suppresses osteogenic differentiation of adult human mesenchymal stem cells. J Cell Biochem. 2004;93:1210-30.

4. Ling L, Nurcombe V, Cool SM. Wnt signaling controls the fate of mesenchymal stem cells. Gene. 2009;433:1-7.

5. Masiakowski P, Carroll RD. A novel family of cell surface receptors with tyrosine kinase-like domain. J Biol Chem. 1992:267:26181-90.

6. Green JL, Kuntz SG, Sternberg PW. Ror receptor tyrosine kinases: orphans no more. Trends Cell Biol. 2008;18:536-44.

7. Stricker S, Rauschenberger V, Schambony A. ROR-family receptor tyrosine kinases. Curr Top Dev Biol. 2017;123:105-42.

8. Stricker S, Mundlos S. FGF and ROR2 receptor tyrosine kinase signaling in human skeletal development. Curr Top Dev Biol. 2011;97:179-206.

9. Liu Y, Rubin B, Bodine PV, Billiard J. Wnt5a induces homodimerization and activation of Ror2 receptor tyrosine kinase. J Cell Biochem. 2008; 105:497-502.

10. Billiard J, Way DS, Seestaller-Wehr LM, Moran RA, Mangine A, Bodine PV. The orphan receptor tyrosine kinase Ror2 modulates canonical Wnt signaling in osteoblastic cells. Mol Endocrinol. 2005:19:90-101.

11. Oishi I, Suzuki H, Onishi N, Takada R, Kani S, Ohkawara B, et al. The receptor tyrosine kinase Ror2 is involved in non-canonical Wnt5a/JNK signalling pathway. Genes Cells. 2003;8:645-54.

12. Mikels AJ, Nusse R. Purified Wnt5a protein activates or inhibits beta-cateninTCF signaling depending on receptor context. PLoS Biol. 2006;4:e115.

13. Schwabe GC, Trepczik B, Süring K, Brieske N, Tucker AS, Sharpe PT, et al. Ror2 knockout mouse as a model for the developmental pathology of autosomal recessive Robinow syndrome. Dev Dyn. 2004;229:400-10.

14. Takeuchi S, Takeda K, Oishi I, Nomi M, Ikeya M, Itoh K, et al. Mouse Ror2 receptor tyrosine kinase is required for the heart development and limb formation. Genes Cells. 2000:5:71-8.

15. Patton MA, Afzal AR. Robinow syndrome. J Med Genet. 2002;39:305-10. 
16. Beiraghi S, Leon-Salazar V, Larson BE, John MT, Cunningham ML, Petryk A, et al. Craniofacial and intraoral phenotype of Robinow syndrome forms. Clin Genet. 2011;80:15-24.

17. Liu Y, Bhat RA, Seestaller-Wehr LM, Fukayama S, Mangine A, Moran RA, et al. The orphan receptor tyrosine kinase Ror2 promotes osteoblast differentiation and enhances ex vivo bone formation. Mol Endocrinol. 2007; 21:376-87.

18. Liu Y, Bodine PV, Billiard J. Ror2, a novel modulator of osteogenesis. J Musculoskelet Neuronal Interact. 2007;7:323-4.

19. Martin JF, Olson EN. Identification of a prx1 limb enhancer. Genesis. 2000;26: 225-9.

20. Logan M, Martin JF, Nagy A, Lobe C, Olson EN, Tabin CJ. Expression of Cre recombinase in the developing mouse limb bud driven by a Prxl enhancer. Genesis. 2002;33:77-80.

21. Branda CS, Dymecki SM. Talking about a revolution: the impact of sitespecific recombinases on genetic analyses in mice. Dev Cell. 2004;6:7-28.

22. Kim H, Kim M, Im SK, Fang S. Mouse Cre-LoxP system: general principles to determine tissue-specific roles of target genes. Lab Anim Res. 2018;34:147-59.

23. Ho HY, Susman MW, Bikoff JB, Ryu YK, Jonas AM, Hu L, et al. Wnt5a-RorDishevelled signaling constitutes a core developmental pathway that controls tissue morphogenesis. Proc Natl Acad Sci U S A. 2012;109:4044-51.

24. Li J. JAK-STAT and bone metabolism. JAKSTAT. 2013;2:e23930.

25. Abroun S, Saki N, Ahmadvand M, Asghari F, Salari F, Rahim F. STATs: an old story, yet mesmerizing. Cell J. 2015;17:395-411.

26. Galoczova $M$, Coates $P$, Vojtesek B. STAT3, stem cells, cancer stem cells and p63. Cell Mol Biol Lett. 2018;23:12.

27. Itoh S, Udagawa N, Takahashi N, Yoshitake F, Narita H, Ebisu S, et al. A critical role for interleukin-6 family-mediated Stat3 activation in osteoblast differentiation and bone formation. Bone. 2006;39:505-12.

28. Zhou H, Newnum AB, Martin JR, Li P, Nelson MT, Moh A, et al. Osteoblast/ osteocyte-specific inactivation of Stat3 decreases load-driven bone formation and accumulates reactive oxygen species. Bone. 2011;49:404-11.

29. Veskimäe K, Scaravilli M, Niininen W, Karvonen $H$, Jaatinen S, Nykter M, et al. Expression analysis of platinum sensitive and resistant epithelial ovarian cancer patient samples reveals new candidates for targeted therapies. Transl Oncol. 2018:11:1160-70.

30. Fukuyo S, Yamaoka K, Sonomoto K, Oshita K, Okada Y, Saito K, et al. IL-6accelerated calcification by induction of ROR2 in human adipose tissuederived mesenchymal stem cells is STAT3 dependent. Rheumatology (Oxford). 2014:53:1282-90.

31. Rigueur D, Lyons KM. Whole-mount skeletal staining. Methods Mol Biol. 2014;1130:113-21.

32. Bailey S, Karsenty G, Gundberg C, Vashishth D. Osteocalcin and osteopontin influence bone morphology and mechanical properties. Ann N Y Acad Sci. 2017;1409:79-84.

33. Akiyama H, Chaboissier MC, Martin JF, Schedl A, de Crombrugghe B. The transcription factor Sox9 has essential roles in successive steps of the chondrocyte differentiation pathway and is required for expression of Sox5 and Sox6. Genes Dev. 2002;16:2813-28.

34. Akiyama H, Kim JE, Nakashima K, Balmes G, Iwai N, Deng JM, et al. Osteochondroprogenitor cells are derived from Sox9 expressing precursors. Proc Natl Acad Sci U S A. 2005;102:14665-70

35. Komori T. Molecular Mechanism of Runx2-Dependent Bone Development Mol Cells. 2020;43:168-75.

36. Cai SX, Liu AR, He HL, Chen $\mathrm{QH}$, Yang Y, Guo FM, et al. Stable genetic alterations of $\beta$-catenin and Ror2 regulate the Wnt pathway, affect the fate of MSCs. J Cell Physiol. 2014;229:791-800.

37. Nemoto E, Ebe Y, Kanaya S, Tsuchiya M, Nakamura T, Tamura M, et al. Wnt5a signaling is a substantial constituent in bone morphogenetic protein-2-mediated osteoblastogenesis. Biochem Biophys Res Commun. 2012:422:627-32

38. Kim S, Koga T, Isobe M, Kern BE, Yokochi T, Chin YE, et al. Stat1 functions as a cytoplasmic attenuator of Runx2 in the transcriptional program of osteoblast differentiation. Genes Dev. 2003:17:1979-91.

39. Tajima K, Takaishi H, Takito J, Tohmonda T, Yoda M, Ota N, Kosaki N, et al. Inhibition of STAT1 accelerates bone fracture healing. J Orthop Res. 2010;28: 937-41

40. Yamada A, Takami M, Kawawa T, Yasuhara R, Zhao B, Mochizuki A, et al. Interleukin-4 inhibition of osteoclast differentiation is stronger than that of interleukin-13 and they are equivalent for induction of osteoprotegerin production from osteoblasts. Immunology. 2007;120:573-9.
41. Zare F, Dehghan-Manshadi M, Mirshafiey A. The signal transducer and activator of transcription factors lodge in immunopathogenesis of rheumatoid arthritis. Reumatismo. 2015;67:127-37.

42. Hossein G, Arabzadeh S, Salehi-Dulabi Z, Dehghani-Ghobadi Z, Heidarian Y, Talebi-Juybari M. Wnt5A regulates the expression of ROR2 tyrosine kinase receptor in ovarian cancer cells. Biochem Cell Biol. 2017;95:609-15.

43. Mohr A, Chatain N, Domoszlai T, Rinis N, Sommerauer M, Vogt M, et al. Dynamics and non-canonical aspects of JAKSSTAT signalling. Eur J Cell Biol. 2012:91:524-32.

44. Villarroel A, Del Valle-Pérez B, Fuertes G, Curto J, Ontiveros N, Garcia de Herreros A, et al. Src and Fyn define a new signaling cascade activated by canonical and non-canonical Wnt ligands and required for gene transcription and cell invasion. Cell Mol Life Sci. 2020;77:919-35.

\section{Publisher's Note}

Springer Nature remains neutral with regard to jurisdictional claims in published maps and institutional affiliations.
Ready to submit your research? Choose BMC and benefit from:

- fast, convenient online submission

- thorough peer review by experienced researchers in your field

- rapid publication on acceptance

- support for research data, including large and complex data types

- gold Open Access which fosters wider collaboration and increased citations

- maximum visibility for your research: over $100 \mathrm{M}$ website views per year

At $\mathrm{BMC}$, research is always in progress.

Learn more biomedcentral.com/submissions 